\title{
Synthesis of Novel Multifluorinated Pyrazolone-5-one Derivatives via Conventional and Non-conventional Methods
}

\author{
Amol Gadakh (Corresponding author) \\ P. G. Department of Chemistry, New Arts, Science and Commerce \\ College, Ahmednagar, University of Pune, Pune- 414003, India \\ Tel: 91-2852-1314 E-mail: gadakhamol@yahoo.com \\ Chetan Pandit \\ Aurigene Discovery Technologies Ltd, 39-40, KIADB Industrial area \\ Electronic city, Phase-2, Hosur Road, Bangalore-560100 \\ Tel: 91-2852-1314 E-mail: Chetan_p@aurigene.com \\ Sahebrao S. Rindhe \\ P. G. Department of Chemistry, New Arts, Science and Commerce \\ College, Ahmednagar, University of Pune, Pune- 414003, India \\ Tel: 91-241-243-1895 E-mail: rindhe_ss@yahoo.com \\ Bhausaheb Karale \\ Department of Chemistry Radhabai Kale mahila Mahavidyalaya \\ Ahmednagar, University of Pune, Pune-411003 \\ E-mail: bkkarale@yahoo.com
}

\begin{abstract}
An efficient synthesis of 3-trifluoromethyl-1-(3,4-difluorophenyl)-1H-pyrazol-5(4H)-one (3) and their Knoevenagel condensation reaction with 1,4-diphenyl -1H-pyrazole-3-carbaldehydes 4, 4-oxo-4H-chromene-3-carbaldehydes 6 and 2-chloroquinoline-3-carbaldehydes 8 have been described by using conventional and non-conventional techniques. Comparison of conventional and non-conventional techniques like Microwave, Ultrasonic assisted reactions showed that, the later procedure require shorter reaction time, good yield and was applicable for larger set of substrates emphasizing the importance of eco-friendly conditions.
\end{abstract}

Keywords: Pyrazol-5(4H)-one, Pyrazoles, Chromones, Quinolines, Knoevenagel condensations

\section{Introduction}

Pyrazolone are very important class of heterocycles due to their wide applications in pharmacological and biological activities (Arnost et al. 2010; Gursoy et al. 2000; Venkat Ragavan et al. 2009). Large numbers of 2-pyrazolin-5-ones have been used as therapeutics agents such as analgesics and antipyretics, (Himly et al. 2003). It is also well known that they exhibit varied biological activities like antiinflammatory, antibacterial, antifungal, etc. (Karale et al. 2000; Rena et al. 2005).

The chemistry of chromones and its derivatives received considerable attention as it was found readily in natural products, which exhibit antimicrobial activities (Deng et al. 2000; Khan et al 2000). Also, quinoline moiety is of great importance to the chemists as well as biologists as it is found in a large variety of naturally occurring compounds and also chemically useful molecules having diverse biological activities (Nasveld et al. 2005; Denny et al. 2006; Mohamoud et al. 2006; Muruganantham et al. 2004). In the view of various therapeutic aspects of introduction of fluorine in various drugs and biologically active heterocycles, we have decided to incorporate the $\mathrm{CF}_{3}$ and fluorine group in various positions of pyrazolinones to enhance their reactivity. 
In recent years due to increasing environmental awareness in chemical and industrial research, the challenge for a clean environment calls for best procedures like reducing pollution, hazardous byproduct formations.

Keeping a perception on above problems, Microwave and Ultrasound assisted techniques are found to be more effective from environmental as well as from shorter reaction time, high yields, ease of work-up and isolation of products (Maruthikumar et al. 2005; Dalvi et al. 2004; Chornous et al. 2004; Breeuwer et al. 2003; Mojtahedi et al. 2008). Compared with traditional methods, the organic reaction can be easily controlled, more convenient, with higher yield, shorter reaction time or milder conditions under ultrasound irradiation.

Keeping in view of demerits of conventional techniques, we report the synthesis of fluorinated pyrazol -5-one 3 and their Knoevenagel condensations reaction with 1, 4-diphenyl-1 $H$-pyrazole-3-carbaldehyde 4, 4-oxo-4H-chromene-3-carbaldehydes $\mathbf{6}$ and 2-chloroquinoline-3-carbaldehydes 8 using microwave and Ultrasonic techniques.

\section{Result and Discussion}

In the present work we have synthesized 3-trifluoromethyl-1-(3, 4-difluorophenyl)-1 $H$-pyrazol-5(4H) -one 3 by reacting 3, 4-difluorophenyl hydrazine 1 and ethyl 4, 4, 4-trifluoro-3-oxobutanoate $\mathbf{2}$ at $130-135^{\circ} \mathrm{C}$ for $3 \mathrm{~h}$ with constant stirring (Scheme-1).Knoevenagel condensation of 3-trifluoromethyl-1-(3, 4-dilfluorophenyl)-1 $H$-pyrazol-5(4H)-one $\mathbf{3}$ with various Substituted Carbaldehydes like 1,4-diphenyl -1H-pyrazole-3-carbaldehydes 4, 4-oxo- $4 H$-chromene-3-carbaldehydes $\mathbf{6}$ and 2-chloroquinoline-3-carb aldehydes $\mathbf{8}$ resulted in the formation of 3-(trifluoromethyl)-1-(3,4-difluorophenyl)-4-((1,4-diphenyl-1 $H$-pyrazol-3-yl)methylene)-1 $H$-pyrazol-5(4H)-one $\quad 5, \quad 3$-(trifluoromethyl)-1-(3, $\quad 4$-difluorophenyl)-4-((4 -oxo-4H-chromen-3-yl) methylene)-1H-pyrazol-5(4H)-one 7 and 4-((2-chloroquinolin-3-yl) methylene )-3-(trifluoromethyl)-1-(3,4-difluorophenyl)-1H-pyrazol-5(4H)-one 9 with a yield of $68-79 \%$ using conventional and $82-92 \%$ of yields using nonconventional methods (Scheme-2). The exact regioisomerism we could not interpreted for all the obtained products due to multiple fluorine couplings and the complexicity pattern in the ${ }^{1} \mathrm{H}-\mathrm{NMR}$.

The characterization data of the newly synthesized compounds were given in (Table-1).The reaction reported here by using ultrasonication and microwave techniques are superior in terms of higher yields and rapid, environmental friendly, compared to conventional techniques.

\section{Experimental}

\subsection{General}

All the recorded melting points were determined on electro thermal melting point apparatus and are uncorrected. IR spectra were recorded on Shimadzu-470 infrared spectrophotometer. The ${ }^{1} \mathrm{H}-\mathrm{NMR}$ spectra were recorded on Bruker- AMX $300 \mathrm{MHz}$ instrument in $\left(\mathrm{CD}_{3}\right)_{2} \mathrm{SO}$ and TMS as an internal standard. ${ }^{13} \mathrm{C}$ NMR spectra were recorded on $75 \mathrm{MHz}$ with TMS as an internal standard. ${ }^{19} \mathrm{~F}$ NMR were recorded on $282 \mathrm{MHz}$. Microwave Initiator-02 Biotage (700Watt) was used to carry out the reactions. The ultrasonic cleaner from Madhu Lab Tech (Tensil India) $230 \mathrm{~V}, 50 \mathrm{~Hz} 10006 \mathrm{H}$ used to carry out sonication reactions. The purity of compounds was confirmed by thin layer chromatography using Merck silica gel $60 \mathrm{~F}_{254}$ coated aluminium plates.

\subsection{Synthesis of 3-trifluoromethyl-1-(3,4-difluorophenyl)-1H-pyrazol-5(4H)-one (3)}

3, 4-Difluorophenyl hydrazine 1 (10g, $69 \mathrm{mmol})$ and ethyl 4, 4, 4-trifluoro-3-oxobutanoate 2 (12.77g, $69 \mathrm{mmol})$ were mixed in a round bottom flask and heated to $130-135^{\circ} \mathrm{C}$ for $3 \mathrm{~h}$ with constant stirring. After completion of reaction, cooled the reaction mass and triturated with diethyl ether $(25 \mathrm{ml})$. The solid residue was stirred for 5-10min and then filtered and dried to obtain 3-trifluoromethyl-1-(3, 4-difluorophenyl)-1 $H$-pyrazol-5(4H)-one as an off white solid powder $\left(16.48 \mathrm{~g}, 90 \%\right.$ yield, $\left.\mathrm{mp} 186^{\circ} \mathrm{C}\right)$.

${ }^{1} \mathrm{H}-\mathrm{NMR}\left(300 \mathrm{MHz},\left(\mathrm{CD}_{3}\right)_{2} \mathrm{SO}\right) ; \delta 6.0(\mathrm{~s}, 1 \mathrm{H}, \mathrm{CH}), 7.6$ (m, 2H, Ar-H), 7.8 (m, 1H, Ar-H), 13.0 (brs, 1H, enol $\mathrm{OH})$.

${ }^{19} \mathrm{~F}$ NMR $\left(282 \mathrm{MHz},\left(\mathrm{CD}_{3}\right)_{2} \mathrm{SO}\right) ; \delta-57.98,-131.99,-135.74$

${ }^{13} \mathrm{C}$ NMR $\left(75 \mathrm{MHz},\left(\mathrm{CD}_{3}\right)_{2} \mathrm{SO}\right) ; \delta$ 85.82( $\left.\underline{\mathrm{CH}}\right), 111.67\left(\underline{\mathrm{CF}}_{3}\right), 118.62(\mathrm{Ar}-\underline{\mathrm{C}} \mathrm{H}), 123.08(\mathrm{Ar}-\underline{\mathrm{CH}}), 134.60(\mathrm{Ar}-\underline{\mathrm{CH}})$, 141.37(Ar- $\underline{\mathrm{CH}}), 146.70(\mathrm{Ar}-\underline{\mathrm{CH}}), 147.75(\mathrm{Ar}-\underline{\mathrm{CH}}), 149.96(\mathrm{Ar}-\underline{\mathrm{C}} \mathrm{H}), 151.01(\underline{\mathrm{C}} \mathrm{H}-\mathrm{OH})$

Mass $\mathrm{m} / \mathrm{z}: 265\left(\mathrm{M}^{+}\right)$

IR (KBr, cm-1); 3010 (=C-H aromatic stretch), 1619 ( $\mathrm{C}=\mathrm{O}$ cyclic lactum), $1599(\mathrm{C}=\mathrm{N}$ stretch).

3.3 General methods of synthesis of 3-(trifluoromethyl)-1-(3, 4-difluorophenyl)-4-((1, 4-diphenyl-1H-

pyrazol-3-yl)methylene-1H-pyrazol-5(4H)-one derivatives 5a-d, 3-(trifluoromethyl) -1-(3,4- 
dilfluorophenyl)-4-((4-oxo-4H-chromen-3-yl)methylene)-1H-pyrazol-5(4H)-one derivatives $7 a-e \quad$ and 4-((2-chloroquinolin-3-yl)methylene)-3-(trifluoromethyl)-1-(3,4-difluorophenyl)-1H-pyrazol-5(4H)-one derivatives 9 a-e using conventional and non-conventional methods

\subsubsection{Conventional method (A)}

Pyrazolone 3 ( $1 \mathrm{mmol}$ ) and respective carbaldehydes 4, 6, and $\mathbf{8}$ (1 mmol) were taken in ethanol (20-25ml) along with catalytic amount of acetic acid $(0.1 \mathrm{mmol})$ and heated the reaction mass to reflux for about $14-26 \mathrm{~min}$ as mentioned in (Table 1) to obtain respective products. After completion of reaction (monitored by TLC), the reaction mass was allowed to cool to room temperature. The precipitated solid was filtered and dried. Most of the compounds obtained in pure form without recrystallization.

\subsubsection{Microwave method (B)}

Starting material 3 and respective carbaldehyde analogues 4, 6, and $\mathbf{8}$ were taken in equimolar quantities along with catalytic amount of acetic acid in ethanol $(1-2 \mathrm{ml})$ in sealed tube and irradiated for 5 min at temperature $100-125^{\circ} \mathrm{C}$ in Biotage microwave. The progress of reaction was monitored by TLC. After completion of reaction, the reaction mass was allowed to cool to room temperature. The sealed tube was opened and the precipitated solid was filtered out and suck dried. Most of the compounds obtained in pure form without further recrystallization.

\subsubsection{Ultrasounds method (C)}

Both 3 and the respective starting carbaldehyde analogues 4, 6 and 8 were taken in equimolar quantities along with catalytic amount of acetic acid in ethanol $(5 \mathrm{ml})$ in R.B. flask and reaction mass was subjected to ultrasonication for $10 \mathrm{~min}$. The reaction temperature was monitored. After completion of reaction, precipitated solid was filtered and dried. Most of the compounds obtained in pure form without recrystallization. Some of the product which was not pure recrystallized from acetic acid as a solvent.

In conclusion, we have designed and synthesized a new series of novel multifluorinated pyrazolone-5-one derivatives using ultrasonicated and microwave assisted techniques and are more convenient, environmentally safe as they require less volume of solvent, short reaction span and better yields as compared to conventional techniques.

The physical and spectral data of all the synthesized compounds 3-(trifluoromethyl)-1-(3,4-difluoro phenyl)-4-((1,4-diphenyl-1H-pyrazol-3-yl)methylene-1H-pyrazol-5(4H)-one derivatives 5a-d, 3-(trifluoromethyl)-1-(3,4-difluorophenyl)-4-((4-oxo-4H-chromen-3-yl)methylene)-1H-pyrazol-5(4H)-one

derivatives 7a-e and 4-((2-chloroquinolin-3-yl)methylene)-3-(trifluoromethyl)-1- (3, 4-difluorophenyl)-1H-pyrazol-5(4H)-one derivatives 9 a-e are as follows.

4-((4-(2,4-Dichloro-5-fluorophenyl)-1-phenyl-1H-pyrazol-3-yl-methylene)-3-(trifluoromethyl)-1-(3, difluorophenyl)-1H-pyrazol-5 (4H)-one (5a) :

Orange solid, mp $223-224^{\circ} \mathrm{C} .{ }^{1} \mathrm{H}-\mathrm{NMR}\left(300 \mathrm{MHz},\left(\mathrm{CD}_{3}\right)_{2} \mathrm{SO}\right) \delta 7.4(\mathrm{~s}, 1 \mathrm{H}, \mathrm{CH}), 7.5(\mathrm{~m}, 1 \mathrm{H}, \mathrm{Ar}-\mathrm{H}), 7.6-7.7(\mathrm{~m}$, 4H, Ar-H), 7.9 (d, $J=3 \mathrm{~Hz}, 1 \mathrm{H}, \mathrm{Ar}-\mathrm{H}), 8.0$ (m, 3H, Ar-H), 8.2 (d, $J=6 \mathrm{~Hz}, \mathrm{Ar}-\mathrm{H}), 10.2$ (s, 1H, pyrazole ring), IR $(\mathrm{KBr}) v \mathrm{~cm}^{-1} 3001(=\mathrm{C}-\mathrm{H}$ aromatic stretch), $1624(\mathrm{C}=\mathrm{O}$ cyclic lactum $), 1576(\mathrm{C}=\mathrm{N}$ stretch$)$.

4-((4- (4-Ethylphenyl)-1-phenyl-1H-pyrazol-3-yl-methylene)-3-(trifluoromethyl)-1-(3,4-difluorophenyl

)-1H-pyrazol-5 (4H)-one (5b):

Orange solid, mp $197-199^{\circ} \mathrm{C} .{ }^{1} \mathrm{H}-\mathrm{NMR}\left(300 \mathrm{MHz},\left(\mathrm{CD}_{3}\right)_{2} \mathrm{SO}\right) \delta 1.28\left(\mathrm{t}, J=7.7 \mathrm{~Hz}, 3 \mathrm{H}, \mathrm{CH}_{3}\right), 2.8(\mathrm{q}, 2 \mathrm{H}, J=7.3 \mathrm{~Hz}$, $\mathrm{CH}_{2}$ ), 7.4-7.5 (m, 3H, Ar-H), 7.5-7.6 (m, 5H, Ar-H), 7.7 (m, 2H, Ar-H), 8.0 (m, 3H, Ar-H), 10.1 (s, 1H, pyrazole ring ), $\mathrm{IR}(\mathrm{KBr}) v \mathrm{~cm}^{-1} 3010(=\mathrm{C}-\mathrm{H}$ aromatic stretch), $1619(\mathrm{C}=\mathrm{O}$ cyclic lactum $), 1598(\mathrm{C}=\mathrm{N}$ stretch$)$.

3-(Trifluoromethyl)-1-(3, 4-difluorophenyl)-4-((1, 4-diphenyl-1H-pyrazol-3-yl) methylene)-1H-pyraz

ole-5(4H)-one (5c):

Orange solid, mp 208-209 ${ }^{\circ} \mathrm{C} .{ }^{1} \mathrm{H}-\mathrm{NMR}\left(300 \mathrm{MHz},\left(\mathrm{CD}_{3}\right)_{2} \mathrm{SO}\right) \delta 7.5(\mathrm{~m}, 1 \mathrm{H}, \mathrm{Ar}-\mathrm{H}), 7.6-7.8(\mathrm{~m}, 10 \mathrm{H}, \mathrm{Ar}-\mathrm{H})$, 7.9-8.0 (m, 3H, Ar-H), 10.2 (s, 1H, pyrazole ring), IR (KBr) $v \mathrm{~cm}^{-1} 3011(=\mathrm{C}-\mathrm{H}$ aromatic stretch), $1688(\mathrm{C}=\mathrm{O}$ cyclic lactum), $1595(\mathrm{C}=\mathrm{N}$ stretch).

4-((4-(4-Chlorophenyl)-1-phenyl-1H-pyrazol-3-yl-methylene)-3-(trifluoromethyl)-1-(3,4-difluorophenyl)-1H-py razol-5 (4H)-one $(\mathbf{5 d})$ :

Orange solid, mp $235-237^{\circ} \mathrm{C} .{ }^{1} \mathrm{H}-\mathrm{NMR}\left(300 \mathrm{MHz},\left(\mathrm{CD}_{3}\right)_{2} \mathrm{SO}\right) \delta$ 7.4-7.5 (m, 2H, Ar-H), 7.6 (m, 1H, Ar-H), 7.6-7.7 $(\mathrm{m}, 6 \mathrm{H}, \mathrm{Ar}-\mathrm{H}), 7.9-8.0(\mathrm{~m}, 3 \mathrm{H}, \mathrm{Ar}-\mathrm{H}), 10.0\left(\mathrm{~s}, 1 \mathrm{H}\right.$, pyrazole ring), IR (KBr) $v \mathrm{~cm}^{-1} 3100(=\mathrm{C}-\mathrm{H}$ aromatic stretch), 
$1638(\mathrm{C}=\mathrm{O}$ cyclic lactum $), 1590(\mathrm{C}=\mathrm{N}$ stretch $)$.

4-((5, 7-Dichloro-4-oxo-4H-chromen-3-yl)-methylene)-3(trifluoromethyl)-1-(3, 4-difluorophenyl)-1H-

Pyrazol-5 (4H)-one (7a):

Red solid, mp 248-251 ${ }^{\circ} \mathrm{C} .{ }^{1} \mathrm{H}-\mathrm{NMR}\left(300 \mathrm{MHz}, \mathrm{CDCl}_{3}\right) \delta 7.2(\mathrm{~m}, 1 \mathrm{H}, \mathrm{Ar}-\mathrm{H}), 7.7$ (m, 1H), $7.8(\mathrm{~m}, 2 \mathrm{H}, \mathrm{Ar}-\mathrm{H}), 8.2$ $(\mathrm{d}, J=2.3 \mathrm{~Hz}, 1 \mathrm{H}, \mathrm{Ar}-\mathrm{H}), 8.4(\mathrm{~s}, 1 \mathrm{H},=\mathrm{C} \underline{\mathrm{H}}), 10.8\left(\mathrm{~s}, 1 \mathrm{H}, \mathrm{C} \underline{\mathrm{H}}\right.$-chromone ring), IR $(\mathrm{KBr}) v \mathrm{~cm}-{ }^{1} 3062(=\mathrm{C}-\mathrm{H}$ aromatic stretch), $1670(\mathrm{C}=\mathrm{O}$ cyclic lactum $), 1639(\mathrm{C}=\mathrm{O}), 1546(\mathrm{C}=\mathrm{N}$ stretch $)$,

4-((6-Chloro-7-methyl-4-oxo-4H-chromen-3-yl)-methylene)-3(trifluoromethyl)-1-(3,4-difluorophenyl)-1H-pyraz ol-5 (4H)-one (7b):

Black solid, mp 239-241 ${ }^{\circ} \mathrm{C} .{ }^{1} \mathrm{H}-\mathrm{NMR}\left(300 \mathrm{MHz},\left(\mathrm{CD}_{3}\right)_{2} \mathrm{SO}\right) \delta 2.4\left(\mathrm{~s}, 3 \mathrm{H}, \mathrm{CH}_{3}\right), 7.0(\mathrm{~d}, \mathrm{~J}=8.8 \mathrm{~Hz}, 1 \mathrm{H}, \mathrm{Ar}-\mathrm{H}), 7.8$ $(\mathrm{m}, 1 \mathrm{H}, \operatorname{Ar}-\mathrm{H}), 7.9(\mathrm{~m}, 1 \mathrm{H}, \operatorname{Ar}-\mathrm{H}), 8.1(\mathrm{~m}, 1 \mathrm{H}, \operatorname{Ar}-\mathrm{H}), 8.4(\mathrm{~m}, 1 \mathrm{H}, \operatorname{Ar}-\mathrm{H}), 9.1(\mathrm{~s}, 1 \mathrm{H},=\mathrm{C} \underline{\mathrm{H}}), 12.0(\mathrm{~s}, 1 \mathrm{H}$, $\mathrm{CH}$-chromone ring), IR (KBr) $\vee \mathrm{cm}^{-1} 3004(=\mathrm{C}-\mathrm{H}$ aromatic stretch), $1656(\mathrm{C}=\mathrm{O}$ cyclic lactum $), 1627(\mathrm{C}=\mathrm{O})$, $1569(\mathrm{C}=\mathrm{N}$ stretch$)$.

4-((6-Chloro-4-oxo-4H-chromen-3-yl)-methylene)-3(trifluoromethyl)-1-(3,4-difluorophenyl)-1H-pyrazol-5 $(4 \mathrm{H})$-one $(7 \mathbf{c})$ :

Green solid, mp 256-257 ${ }^{\circ} \mathrm{C} .{ }^{1} \mathrm{H}-\mathrm{NMR}\left(300 \mathrm{MHz},\left(\mathrm{CD}_{3}\right)_{2} \mathrm{SO}\right) \delta 7.0(\mathrm{~d}, J=8.8 \mathrm{~Hz}, 1 \mathrm{H}, \mathrm{Ar}-\mathrm{H}), 7.5(\mathrm{~m}, 1 \mathrm{H}, \mathrm{Ar}-\mathrm{H})$, 7.6 - $7.7(\mathrm{~m}, 2 \mathrm{H}, \mathrm{Ar}-\mathrm{H}$, ) , 7.8-7.9 (m, $2 \mathrm{H}, \mathrm{Ar}-\mathrm{H}), 8.1(\mathrm{~m}, 1 \mathrm{H}, \mathrm{Ar}-\mathrm{H}), 10.4$ (s, $1 \mathrm{H}, \mathrm{CH}$-chromone ring), IR (KBr) v $\mathrm{cm}^{-1} 3074$ (=C-H aromatic stretch), $1688(\mathrm{C}=\mathrm{O}$ cyclic lactum $), 1668(\mathrm{C}=\mathrm{O}), 1645(\mathrm{C}=\mathrm{N}$ stretch $)$.

3-(Trifluoromethyl)-1-(3, 4-difluorophenyl)-4-((6, 8-dimethyl-4-oxo-4H-chromen-3-yl)-methylene)-1H

-Pyrazol-5 (4H)-one (7d):

Red solid, mp 264-265 ${ }^{\circ} \mathrm{C} .{ }^{1} \mathrm{H}-\mathrm{NMR}\left(300 \mathrm{MHz}, \mathrm{CDCl}_{3}\right) \delta 2.4\left(\mathrm{~s}, 3 \mathrm{H}, \mathrm{CH}_{3}\right), 2.5\left(\mathrm{~s}, 3 \mathrm{H}, \mathrm{CH}_{3}\right), 7.2(\mathrm{~s}, 1 \mathrm{H}, \mathrm{Ar}-\mathrm{H})$, $7.4(\mathrm{~s}, 1 \mathrm{H}, \mathrm{Ar}-\mathrm{H}), 7.7$ (m, 1H, Ar-H), $7.9(\mathrm{~m}, 2 \mathrm{H}, \mathrm{Ar}-\mathrm{H}), 8.4(\mathrm{~s}, 1 \mathrm{H},=\mathrm{C} \underline{\mathrm{H}}), 10.8$ (s, 1H, C트-chromone ring), IR $(\mathrm{KBr}) v \mathrm{~cm}^{-}{ }^{1} 3049(=\mathrm{C}-\mathrm{H}$ aromatic stretch), $1694(\mathrm{C}=\mathrm{O}$ cyclic lactum $), 1655(\mathrm{C}=\mathrm{O}), 1612(\mathrm{C}=\mathrm{N}$ stretch$)$.

3-(Trifluoromethyl)-1-(3,4-difluorophenyl)-4-((7-methyl-4-oxo-4H-chromen-3-yl)-methylene)-1H-pyrazol-5 (4H)-one (7e):

Red solid, mp $243-244^{\circ} \mathrm{C} .{ }^{1} \mathrm{H}-\mathrm{NMR}\left(300 \mathrm{MHz},\left(\mathrm{CD}_{3}\right)_{2} \mathrm{SO}\right) \delta 2.5\left(\mathrm{~s}, 3 \mathrm{H}, \mathrm{CH}_{3}\right), 7.2(\mathrm{~m}, 1 \mathrm{H}, \mathrm{Ar}-\mathrm{H}), 7.4(\mathrm{~m}, 1 \mathrm{H}$, $\operatorname{Ar}-\mathrm{H}), 7.5(\mathrm{~m}, 1 \mathrm{H}, \mathrm{Ar}-\mathrm{H}), 7.6(\mathrm{~m}, 1 \mathrm{H}, \mathrm{Ar}-\mathrm{H}), 7.8(\mathrm{~m}, 1 \mathrm{H}, \mathrm{Ar}-\mathrm{H}), 8.1(\mathrm{~s}, 1 \mathrm{H},=\mathrm{CH}), 8.2(\mathrm{~d}, J=7.9 \mathrm{~Hz}, 1 \mathrm{H}), 10.8$ (s, $1 \mathrm{H}, \mathrm{CH}$-chromone ring), IR $(\mathrm{KBr}) \vee \mathrm{cm}^{-1} 3008(=\mathrm{C}-\mathrm{H}$ aromatic stretch), $1678(\mathrm{C}=\mathrm{O}$ cyclic lactum), 1635 $(\mathrm{C}=\mathrm{O}), 1600(\mathrm{C}=\mathrm{N}$ stretch $)$.

4-((2-Chloro-6-methoxyquinolin-3-yl)-methylene)-3-(trifluoromethyl)-1-(3,4-dilfuorophenyl)-1H-pyrazol-5(4H) -one (9a):

Black solid, mp $278-279^{\circ} \mathrm{C} .{ }^{1} \mathrm{H}-\mathrm{NMR}\left(300 \mathrm{MHz},\left(\mathrm{CD}_{3}\right)_{2} \mathrm{SO}\right) \delta 4.0\left(\mathrm{~s}, 3 \mathrm{H}, \mathrm{OCH}_{3}\right), 6.0(\mathrm{~s}, 1 \mathrm{H},=\mathrm{C} \underline{\mathrm{H}}), 7.2(\mathrm{dd}, J=$ $9.0 \& 2.0 \mathrm{~Hz}, 1 \mathrm{H}, \mathrm{Ar}-\mathrm{H}), 7.4(\mathrm{~d}, J=2 \mathrm{~Hz}, 1 \mathrm{H}, \mathrm{Ar}-\mathrm{H}), 7.6(\mathrm{~m}, 2 \mathrm{H}, \mathrm{Ar}-\mathrm{H}), 7.8(\mathrm{~m}, 2 \mathrm{H}, \mathrm{Ar}-\mathrm{H}), 8.2(\mathrm{~s}, 1 \mathrm{H}, \mathrm{Ar}-\mathrm{H}), \mathrm{IR}$ $(\mathrm{KBr}) v \mathrm{~cm}-{ }^{1} 3014(=\mathrm{C}-\mathrm{H}$ aromatic stretch $), 1695(\mathrm{C}=\mathrm{O}$ cyclic lactum $), 1661(\mathrm{C}=\mathrm{N}$ stretch $)$,

4-((2-Chloro-6-methylquinolin-3-yl)-methylene)-3-(trifluoromethyl)-1-(3, 4-dilfuorophenyl)-1H-pyraz ol-5(4H)-one (9b):

Orange solid, mp 268-269 ${ }^{\circ} \mathrm{C} .{ }^{1} \mathrm{H}-\mathrm{NMR}\left(300 \mathrm{MHz},\left(\mathrm{CD}_{3}\right)_{2} \mathrm{SO}\right) \delta 2.4\left(\mathrm{~s}, 3 \mathrm{H}, \mathrm{CH}_{3}\right), 6.0(\mathrm{~s}, 1 \mathrm{H},=\mathrm{C} \underline{\mathrm{H}}), 7.15(\mathrm{~s}, 1 \mathrm{H}$, Ar-H), $7.4(\mathrm{~d}, J=6 \mathrm{~Hz}, 1 \mathrm{H}, \mathrm{Ar}-\mathrm{H}), 7.6-7.7(\mathrm{~m}, 2 \mathrm{H}, \mathrm{Ar}-\mathrm{H}), 7.8-8.0$ (m, 2H, Ar-H), 8.3 (s, 1H, Ar-H), IR (KBr) v $\mathrm{cm}^{-1} 3089$ (=C-H aromatic stretch), $1687(\mathrm{C}=\mathrm{O}$ cyclic lactum $), 1651(\mathrm{C}=\mathrm{N}$ stretch $)$.

4-((2-Chloro-8-methylquinolin-3-yl)-methylene)-3-(trifluoromethyl)-1-(3, 4-dilfuorophenyl)-1H-pyraz

Ol-5(4H)-one (9c):

Orange solid, mp 269-272 ${ }^{\circ} \mathrm{C} .{ }^{1} \mathrm{H}-\mathrm{NMR}\left(300 \mathrm{MHz},\left(\mathrm{CD}_{3}\right)_{2} \mathrm{SO}\right) \delta 2.4\left(\mathrm{~s}, 3 \mathrm{H}, \mathrm{CH}_{3}\right), 6.0(\mathrm{~s}, 1 \mathrm{H},=\mathrm{CH}), 7.2(\mathrm{~m}, 1 \mathrm{H}$, Ar-H), 7.5 (m, 1H, Ar-H), 7.6 (m, 2H, Ar-H), $7.7-8.0$ (m, 2H, Ar-H), 8.3 (s, 1H, Ar-H), IR (KBr) $v \mathrm{~cm}^{1}{ }^{1} 3092$ ( $=\mathrm{C}-\mathrm{H}$ aromatic stretch), $1678(\mathrm{C}=\mathrm{O}$ cyclic lactum $), 1650(\mathrm{C}=\mathrm{N}$ stretch).

4-((2-Chloro-7-methylquinolin-3-yl)-methylene)-3-(trifluoromethyl)-1-(3, 4-dilfuorophenyl)-1H-pyraz Ol-5(4H)-one (9d):

Red solid, mp 264-265 ${ }^{\circ} \mathrm{C} .{ }^{1} \mathrm{H}-\mathrm{NMR}\left(300 \mathrm{MHz},\left(\mathrm{CD}_{3}\right)_{2} \mathrm{SO}\right) \delta 2.4\left(\mathrm{~s}, 3 \mathrm{H}, \mathrm{CH}_{3}\right), 6.0(\mathrm{~s}, 1 \mathrm{H},=\mathrm{C} \underline{\mathrm{H}}), 7.6(\mathrm{~m}, 2 \mathrm{H}$, Ar-H), 7.8 (m, 3H, Ar-H), 8.0 (m, 1H, Ar-H), 8.2 (s, 1H,Ar-H), IR (KBr) $v$ cm- ${ }^{1} 3034$ (=C-H aromatic stretch), $1698(\mathrm{C}=\mathrm{O}$ cyclic lactum $), 1646(\mathrm{C}=\mathrm{N}$ stretch $)$. 
4-((2-Chloroquinolin-3-yl)-methylene)-3-(trifluoromethyl)-1-(3,4-dilfuorophenyl)-1H-pyrazol-5(4H)-one (9e):

Red solid, mp 263-265 ${ }^{\circ} \mathrm{C} .{ }^{1} \mathrm{H}-\mathrm{NMR}\left(300 \mathrm{MHz},\left(\mathrm{CD}_{3}\right)_{2} \mathrm{SO}\right) \delta 7.2(\mathrm{~m}, 1 \mathrm{H}, \mathrm{Ar}-\mathrm{H}), 7.4(\mathrm{~m}, 1 \mathrm{H}, \mathrm{Ar}-\mathrm{H}), 7.6-7.7(\mathrm{~m}$, $3 \mathrm{H}, \mathrm{Ar}-\mathrm{H}), 7.8(\mathrm{~m}, 1 \mathrm{H}, \mathrm{Ar}-\mathrm{H}), 7.9(\mathrm{~m}, 1 \mathrm{H}, \mathrm{Ar}-\mathrm{H}), 8.3$ (s, 1H, Ar-H), 10.0 (s, $1 \mathrm{H}, \mathrm{Ar}-\mathrm{H}), \mathrm{IR}(\mathrm{KBr}) v \mathrm{~cm}-{ }^{1} 3045$ $(=\mathrm{C}-\mathrm{H}$ aromatic stretch $), 1678(\mathrm{C}=\mathrm{O}$ cyclic lactum $), 1650(\mathrm{C}=\mathrm{N}$ stretch $)$.

\section{Acknowledgements}

We greatly acknowledge to the C.E.O. Mr. C. S. N. Murthy of Aurigene Discovery Technologies Ltd. for constant encouragement and providing necessary facilities.

\section{References}

63, 1082-1089.

Arnost, M., Pierce, A., Haar, E., Lauffer, D., Madden, J., Tanner, K., Green, J. (2010). 3-Aryl-4-(aryl hydrazano)-1H-pyrazol-5-ones: Highly ligand efficient and potent inhibitors of GSK3ß. Biorg. Med. Chem. Lett., 20, 1661-1664.

Breeuwer, R., Blacquière, G. (2003). Modeling, monitoring and control of continuous wave ultrasonic systems. Ultrason. Sonochem., 10, 197-201.

Chloro chromones and 2-(1, 3-Diphenyl-1H-pyrazol-4-yl)-4-(2-hydroxyphenyl)-2, 3-dihydro-1, 5 benzothiazepines. Korean J. Med. Chem., 10 (2) 84-96.

Chornous, V. O., Bratenko, M. K. \& Vovk, M. V. (2004). Microwave-Assisted Synthesis of 3-(4-Pyrazolyl) propenoic Acids. Synth Commun., 34(1) 79-83.

Dalvi, N. R., Karale, B. K., Gill, C. H. (2004). Environmentally benign synthesis of fluorinated pyrazolone derivatives and their antimicrobial activity. Indian J. chem. 1(8) 582-583.

Deng, Y., Lee, J. P., Ramamonjy, M. T., Snyder, J. K., Des Etages, S. A., Kanada, D., Snyder, M.P., Turner, C. J. (2000). New Antimicrobial flavanones from Physena madagascariensis. J. Nat. Product,

Denny, W. A., Wilson, W. R., Ware, D. C., Atwell, G. J., Milbank, J. B., Stevenson, R. J. (2006). U.S. Patent , 7064117.

Gursoy, A., Demirayak, S., Capan, G., Erol, K., Vural, K. (2000). Synthesis and preliminary evaluation of new 5-pyrazolinone derivatives as analgesic agents. Eur. J. Med. Chem., 35, 359-364.

Himly, M., Jahn-Schmid, B., Pittertschatscher, K., Bohle, B., Grubmayr, K., Ferreira, F., Ebner, H., Ebner, C. (2003). IgE-mediated immediate-type hypersensitivity to the pyrazolone drug Propyphenazone. J. Allergy Clin. Immunol., 111, 882-888.

Karale, B. K., Chavan, V. P., Mane, A. S., Hangarge, R. V., Gill, C. H.\& Shingare, M. S. (2000). Synthesis, Characterization and Antimicrobial Activities of some 2-(1, 3-Diphenyl-1H-pyrazol-4-yl)-3-

Khan, I. A., Avery, M. A., Burandt, C. L., Goins, D. K., Mikell, J. R., Nash, T. E., Azadegan, A., Walker, L. A. (2000). Antigiardial Activity of Isoflavones from Dalbergia frutescens Bark. J. Nat. Product, 63, 1414-1416.

Maruthikumar, T. V., Reddy, V. P. \& Rao, P. H. (2005). Microwave induced solvent - free synthesis of 1-aryl-2-(1E)-arylvinyl-4-arylmethylene-2-imidazolin-5-one. Indian J. chem. 44B, 1931.

Mohamoud, A., Chevalier, J., Davin-Regli, A., Barbe, J., Jean-Marie. (2006). Quinoline Derivatives as Promising Inhibitors of Antibiotic Efflux Pump in Multidrug Resistant Enterobacter Aerogenes Isolates. Current Drug Targets, 7, 843-847.

Mojtahedi, M., Javadpour, M., Abaee, M.S. (2008). Convenient ultrasound mediated synthesis of substituted pyrazolones under solvent- free condition. Ultrason. Sonochem., 15, 828-832.

Muruganantham, N., Sivakumar, R., Anbalagan, N., Ginasekaran., Leonard, J. T. (2004). Synthesis,<smiles>NNc1ccc(F)c(F)c1</smiles>

1

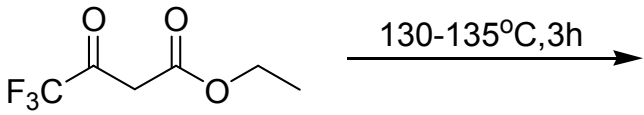

2<smiles>O=C1CC(C(F)(F)F)=NN1c1ccc(F)c(F)c1</smiles>

3 
Anticonvulsant and Antihypertensive Activities of 8-Substituted Quinoline Derivatives. Biol. Pharm. Bull., 27, 1683-1687.

Nasveld, P., Kitchener, S. (2005). Treatment of acute vivax malaria with tafenoquine. Transactions of the Royal Society of Tropical Medicine and Hygiene, 99, 2-5.Leatham, P. A., Bird, H. A., Wright, V., Seymour, D., Gordon, A., (1983). A double blind study of antrafenine, naproxen and placebo in osteoarthritis. Eur. J. Rhematol. Inflamm., 6, 209-211.

Rena, X. L., Hua, B., Yang, H. Z. (2005). Synthesis of small library containing substituted pyrazoles. ARKIVOC, 15, 59-67.

Venkat Ragavan, R., Vijaykumar, V., Suchetha Kumari, N. (2009). Synthesis of some novel bioactive 4 oxy/thio substituted-1H- pyrazol-5(4H)-ones via efficient cross-Claisen condensation. Eur. J. Med. Chem., 44, 3852-3857.

Scheme 1

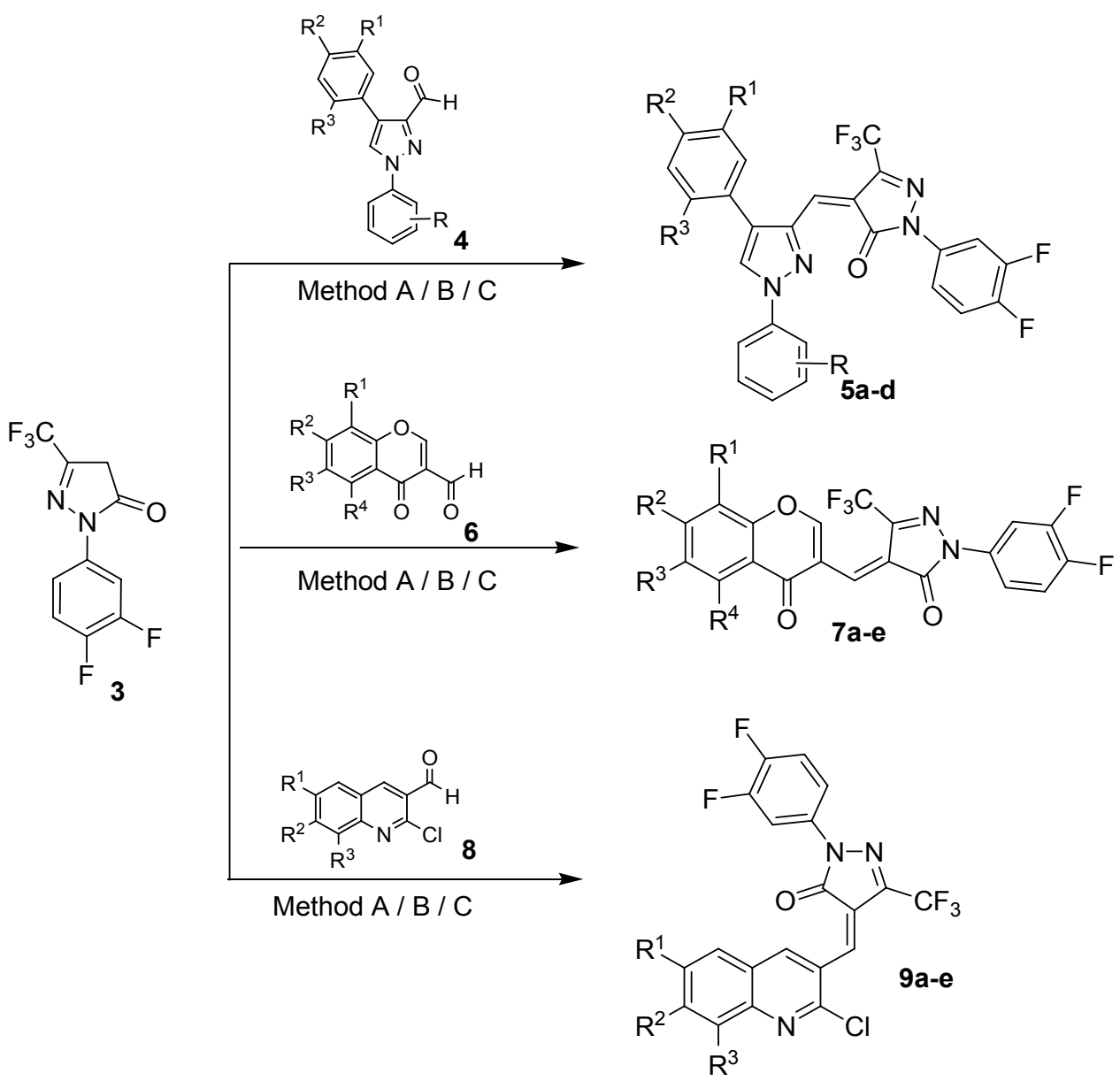

Scheme 2. Method A: EtOH, Cat. AcOH, reflux; Method B: EtOH, Cat. AcOH, Microwave; Method C: EtOH, Cat. AcOH, Sonication 
Table 1. Time and yield data of newly synthesized compounds 5a-d, 7a-e and 9a-e using conventional, non conventional techniques

\begin{tabular}{|c|c|c|c|c|c|c|c|c|}
\hline \multirow{2}{*}{$\begin{array}{l}\text { Compound } \\
\text { no. }\end{array}$} & \multirow[t]{2}{*}{$\mathbf{R}^{1}$} & \multirow[t]{2}{*}{$\mathbf{R}^{2}$} & \multirow[t]{2}{*}{$\mathbf{R}^{3}$} & \multirow[t]{2}{*}{$\mathbf{R}^{4}$} & \multicolumn{2}{|c|}{ Conventional } & \multirow{2}{*}{$\begin{array}{l}\text { MW } \\
(\mathbf{t}=\mathbf{5 m i n})\end{array}$} & \multirow{2}{*}{$\begin{array}{l}\text { US } \\
(\mathbf{t}=\mathbf{1 0 m i n}) \\
\% \text { yield }\end{array}$} \\
\hline & & & & & $\mathrm{t}(\mathrm{min})$ & $\%$ yield & & \\
\hline $5 \mathbf{a}$ & $\mathrm{F}$ & $\mathrm{Cl}$ & $\mathrm{Cl}$ & -- & 15 & 60 & 92 & 77 \\
\hline $5 b$ & $\mathrm{H}$ & Et & $\mathrm{H}$ & -- & 18 & 68 & 95 & 80 \\
\hline $5 c$ & $\mathrm{H}$ & $\mathrm{H}$ & $\mathrm{H}$ & -- & 14 & 67 & 93 & 77 \\
\hline $5 d$ & $\mathrm{H}$ & $\mathrm{Cl}$ & $\mathrm{H}$ & -- & 20 & 69 & 88 & 80 \\
\hline $7 \mathbf{a}$ & $\mathrm{H}$ & $\mathrm{Cl}$ & $\mathrm{H}$ & $\mathrm{Cl}$ & 21 & 71 & 88 & 80 \\
\hline $7 b$ & $\mathrm{H}$ & $\mathrm{CH}_{3}$ & $\mathrm{Cl}$ & $\mathrm{H}$ & 23 & 65 & 88 & 82 \\
\hline $7 \mathrm{c}$ & $\mathrm{H}$ & $\mathrm{H}$ & $\mathrm{Cl}$ & $\mathrm{H}$ & 24 & 68 & 93 & 78 \\
\hline $7 d$ & $\mathrm{CH}_{3}$ & $\mathrm{H}$ & $\mathrm{CH}_{3}$ & $\mathrm{H}$ & 26 & 69 & 89 & 76 \\
\hline $7 e$ & $\mathrm{H}$ & $\mathrm{CH}_{3}$ & $\mathrm{H}$ & $\mathrm{H}$ & 23 & 71 & 93 & 78 \\
\hline $9 \mathbf{a}$ & $\mathrm{OMe}$ & $\mathrm{H}$ & $\mathrm{H}$ & -- & 25 & 66 & 92 & 79 \\
\hline $9 b$ & $\mathrm{CH}_{3}$ & $\mathrm{H}$ & $\mathrm{H}$ & -- & 24 & 59 & 89 & 84 \\
\hline $9 c$ & $\mathrm{H}$ & $\mathrm{H}$ & $\mathrm{CH}_{3}$ & -- & 23 & 71 & 90 & 82 \\
\hline 9d & $\mathrm{H}$ & $\mathrm{CH}_{3}$ & $\mathrm{H}$ & -- & 25 & 70 & 92 & 88 \\
\hline $9 e$ & $\mathrm{H}$ & $\mathrm{H}$ & $\mathrm{H}$ & -- & 23 & 71 & 92 & 83 \\
\hline
\end{tabular}

5a-c $\mathrm{R}=\mathrm{H}$; 5d, $\mathrm{R}=4$-Fluoro; $\mathrm{MW}=$ Microwave, $\mathrm{US}=$ Ultrasound sonication, $\mathrm{t}=$ time 